\title{
BNL-111767-2016-JA
}

\section{Engineering catalytic activity via ion beam bombardment of catalyst supports for vertically aligned carbon nanotube growth}

\author{
A. E. Islam* ${ }^{1,2}$, P. Nikoleav ${ }^{1,3}$, P.B. Amama ${ }^{4}$, D. Zakharov ${ }^{5}$, G. Sargent ${ }^{1,3}$, S. Saber ${ }^{1,3}$, D. \\ Huffman $^{1,3,6}$, M. Erford ${ }^{7}$, S. L. Semiatin ${ }^{1}$, E. A. Stach ${ }^{5}$, and B. Maruyama*1 \\ ${ }^{1}$ Materials and Manufacturing Directorate, Air Force Research Laboratory, Wright-Patterson Air \\ Force Base, OH 45433, USA; ${ }^{2}$ National Research Council, Washington D.C. 20001, USA; \\ ${ }^{3}$ Biological and Nanoscale Technologies Division, UES, Inc., Dayton, OH 45432, USA; \\ ${ }^{4}$ Department of Chemical Engineering, Kansas State University, Manhattan, Kansas 66506, USA; \\ ${ }^{5}$ Center for Functional Nanomaterials, Brookhaven National Laboratory, Upton, NY 11973, USA; \\ ${ }^{6}$ Department of Physics, Wright State University, Dayton, OH 45435, USA; ${ }^{7}$ Southwestern Ohio \\ Council for Higher Education, Dayton, $\mathrm{OH} 45403$
}

\begin{abstract}
Carbon nanotube growth depends on the catalytic activity of metal nanoparticles on alumina or silica supports. The control on catalytic activity is generally achieved by variations in water concentration, carbon feed, and sample placement on a few limited types of alumina or silica catalyst supports obtained via thin film deposition. We have recently expanded the choice of catalyst supports by engineering inactive substrates like c-cut sapphire via ion beam bombardment. The deterministic control on the structure and chemistry of catalyst supports obtained by tuning the degree of beam-induced damage have enabled better regulation of the activity of Fe catalyst only in the ion beam bombarded areas and hence enabled controllable super growth of carbon nanotube. A wide range of surface characterization techniques were used to monitor the catalytically active surface engineered via ion beam bombardment. The proposed method offers a versatile way to control carbon nanotube growth in patterned areas and also enhances the current understanding of the growth process. With the right choice of water concentration, carbon feed and sample placement, engineered catalyst supports may extend the carbon nanotube growth yield to a level that is even higher than the ones reported here, and thus offers promising applications of carbon nanotubes in electronics, heat exchanger, and energy storage.
\end{abstract}

Keywords: Carbon nanotube, catalytic activity, catalyst support, ion beam bombardment.

\section{INTRODUCTION}

Carbon nanotubes (CNTs) are one of the most exciting materials in the recent history. The application of CNTs has been explored in ultra-lightweight composites for aerospace [1-3], electrical cables [4,5], interconnects [6], bio/chemical sensors [7, 8], energy storage devices [9, 10], field-emitting devices [4] and high speed electronics [11-13]. A realization of these applications requires large scale production of CNTs with sufficient control over yield, density and electronic type during growth. A larger CNT yield is desired, in general, for any application [14-17], while certain applications like interconnects require a CNT density of $\sim 10^{13} \mathrm{~cm}^{-2}[6,18]$ and high-performance electronics require a $99.99 \%$ purity in electronic type (semiconducting) [12,13]. To meet these specifications, one must have good control on the catalytic activity of metal nanoparticles used for CNT growth with precise placement of particles that will yield CNTs with a

*aeislam@ieee.org; benji.maruyama@us.af.mil 
desired level of yield, density, and chirality. Despite decades of CNT research and the clear need for improved growth, progress on fundamental understanding of catalytic activity and resultant CNT growth have been limited.

Vertically aligned CNT (VA-CNT) growth generally involves deposition of thin films of metals like Fe, Co, and Ni on a wide variety of catalyst supports $[19,20]$. These films form metal nanoparticles at the temperatures used for VA-CNT growth and the nanoparticles later catalyze CNTs at a low efficiency (10-80\%) [21]. The catalytic activity of nanoparticles on solid supports (heterogeneous catalysis) is affected by water vapor [14, 15, 22] and carbon feedstock.[16] In addition, the catalytic activity is most efficient only on certain types of catalyst supports, specifically alumina or silica [23-27]. Even for materials like alumina or silica, only certain types of alumina $\left(\mathrm{AlO}_{\mathrm{x}}\right)[23,24,28,29]$, quartz [30] or sapphire [31] yield extended catalytic activity and hence longer VA-CNT growth. Our previous study with a variety of alumina supports clarified the role of catalyst support in enhancing catalytic activity [23, 24, 32]. In that study, while pristine sapphire $\left(\mathrm{Al}_{2} \mathrm{O}_{3}\right)$ did not support VA-CNT growth, porous alumina $\left(\mathrm{AlO}_{\mathrm{x}}\right)$ deposited by three different thin-film deposition methods (namely magnetron sputtering, electron beam evaporation, and atomic layer deposition) showed higher catalytic activity and better VA-CNT growth. The key microstructural element for enhancing catalytic activity was porosity of the $\mathrm{AlO}_{\mathrm{x}}$ support that allowed mass loss for the catalyst via sub-surface diffusion and hence restricted coarsening of the catalyst by inhibiting Ostwald ripening [22]. The limited number of techniques for metal film deposition, however, only allowed a few options for catalyst supports [23] and, therefore, limited the exploration of an important process parameters.

In our recent publication discussed in this proceedings article [33], we have presented ion beam bombardment as an effective method to create catalyst supports with controllable degrees of catalyst activity and lifetime for VA-CNT growth. The presented method converts an inactive catalyst support like c-cut sapphire [23,31] into a highly active support with extended catalytic activity and $\sim \mathrm{mm}$ tall VA-CNT growth only in the ion beam bombarded areas. The conversion of catalyst supports and resultant catalytic activity were monitored using well-established surface characterization methods. Degree of nanotube growth on ion beam damaged substrates shows good correlation with the catalytic activity on the engineered catalyst supports. The method expands the choice of catalyst supports, an important component for CNT growth, from a few types of alumina to a wide range of engineered substrates.

\section{EXPERIMENTAL DETAILS}

To convert inactive catalyst supports like c-cut sapphire into an active one, the substrates were bombarded with argon $\left(\mathrm{Ar}^{+}\right)$ions at different accelerating voltages and at different ion doses. The surfaces of bombarded catalyst support were characterized using X-ray photoelectron spectroscopy (XPS) and atomic force microscopy (AFM), while the extent of ion damage was analyzed using cross-sectional TEM (X-TEM) and X-ray reflectivity (XRR). For VA-CNT growth, a thin film $(\sim 1 \mathrm{~nm})$ of Fe was deposited on the ion damaged substrates. Substrates were later annealed at $585{ }^{\circ} \mathrm{C}$ for 10 min and then rapidly cooled down to room temperature, both in hydrogen ambient with $200 \mathrm{sccm}$ flow. Some samples were removed from the reactor at this point to analyze catalyst morphology using Atomic Force Microscopy (AFM). Other samples were immediately subjected to VA-CNT growth at $760{ }^{\circ} \mathrm{C}$ with a mixed flow of argon $(470 \mathrm{sccm})$, hydrogen $(100 \mathrm{sccm})$ and ethylene $(25 \mathrm{sccm})$ for $30 \mathrm{~min}$ and using $\sim 50 \mathrm{ppm}$ water vapor. Grown CNTs were analyzed using scanning electron microscope (SEM), Raman spectroscopy, and transmission electron microscope (TEM).

XPS analysis were performed at $\sim 4 \times 10^{-7} \mathrm{~Pa}$ base pressure using Surface Science Instruments (SSI) M-probe equipped with an Al K $\alpha$ X-ray source and the acquired data was analyzed using CASA XPS software with Shirley background subtraction. AFM was carried out in a Veeco Nanoscope Multimode instrument using MikroMasch HQ:NSC15/Al-BS (having tip radius $\sim 8 \mathrm{~nm}$, resonant frequency $265-410 \mathrm{kHz}$, and force constant 20-80 N/m). TEM studies were performed in a FEI Titan Environmental TEM at an accelerating voltage of $300 \mathrm{kV}$. XRR were carried out in air using Rigaku-Smartlab X-ray diffractometer equipped with a $\mathrm{Cu} \mathrm{K \alpha}(\lambda=0.154 \mathrm{~nm})$ radiation source using slit collimation.

\section{RESULTS AND DISCUSSION}

\section{VA-CNT growth using ion beam bombardment}

Figure 1a shows side view of a VA-CNT grown sapphire substrate. A carpet height of $\sim 0.8 \mathrm{~mm}$ is achieved here via bombardment of $\mathrm{Ar}^{+}$ion accelerated at $5 \mathrm{kV}$ at a dose of $\sim 2.1 \times 10^{20} \mathrm{~cm}^{-2}$. The degree of VA-CNT growth is comparable to that obtained in $\mathrm{ALD}$ or sputter-deposited $\mathrm{AlO}_{\mathrm{x}}$ using lateral gas flow [17]. Raman spectra taken from the top of the carpet (Figure 1b) suggests the presence of disorder within the grown CNTs with a strong D and $\mathrm{G}^{\prime}$ band that have intensities comparable to that of $\mathrm{G}$ band. Low G/D ratio ( 1.4) in Raman spectra is also an indicator about the 
abundance of multi-walled CNTs in the samples [34]. In addition, single-walled CNTs at the top of the carpet with radial breathing mode (RBM) signals in the range of $150-300 \mathrm{~cm}^{-1}$ are also observed. A detailed SEM image taken at the top of VA-CNT carpet shows the presence of flakes (Figure 1c), which have presumably been lifted off from the top of catalyst support during CNT growth. Similar phenomenon has often been observed in VA-CNT literature $[35,36]$ and is termed as "odako" growth [37]. The controllability offered by the ion beam in engineering catalyst supports allows us to obtain different degrees of VA-CNTs either by varying accelerating voltages or by varying damage doses of the $\mathrm{Ar}^{+}$ion. Figure 1d-e shows height of VA-CNTs grown on sapphire surfaces damaged at various degrees of ion beam conditions. Increased degrees of $\mathrm{Ar}^{+}$ion damage, either by increasing the accelerating voltage at a fixed damage dose (Figure 1d) or by increasing the damage dose at a fixed acceleration voltage (Figure 1e), results in higher carpet height. In addition, a patterned VA-CNT growth in Figure if by masking the substrate with a $\mathrm{SiO}_{\mathrm{x}}$-coated Mo TEM grid confirms confinement of catalyst activity within the damaged areas. (Significant amount of flakes at the top of carpet (Figure S2 of supporting information), as in "odako" growth,[35-37] and enhancement of growth near the mask edges[35] are also observed here.) In this substrate with patterned VA-CNT growth, Fe catalyst ( 1 nm Fe film) was uniformly deposited on the entire substrate; however, only the ion damaged regions grew VA-CNTs. In contrast, patterning the catalyst film deposition $[15,38]$ to achieve selective area growth suffers from potential loss of fidelity in the pattern transfer via catalyst migration into regions outside the patterned area.
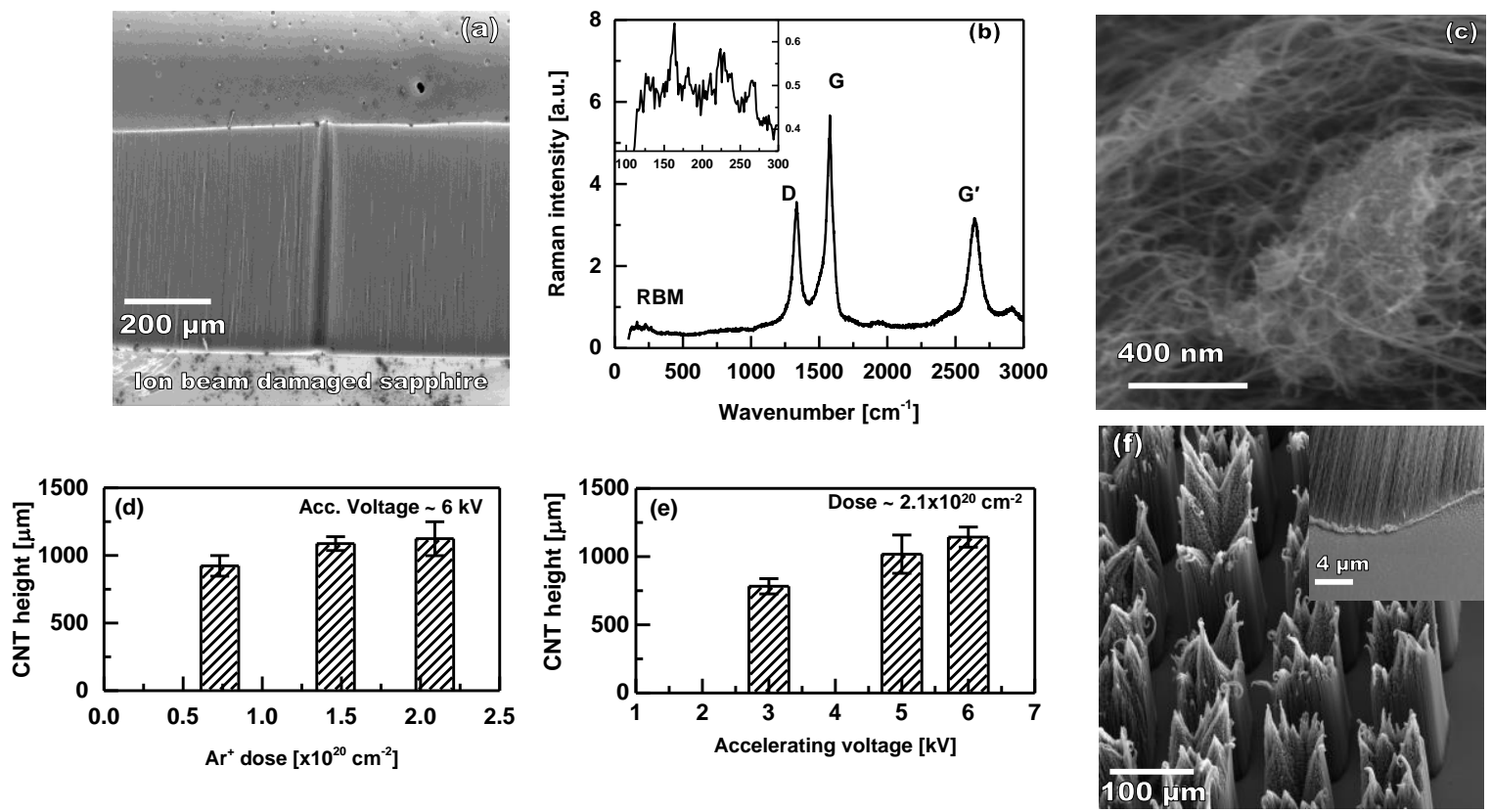

Figure 1. Ion beam bombarded c-cut sapphire substrate supports VA-CNT growth in a controlled manner: (a) SEM images show $\sim 0.8 \mathrm{~mm}$ tall CNT grown on the bombarded substrate. (b) Raman spectra taken from the top of VA-CNT shows the presence of $\mathrm{G}^{\prime}$ band with a G/D ratio of $\sim 1.4$ and with radial breathing modes (RBMs; inset) in the range of $150-300 \mathrm{~cm}^{-1}$. (c) SEM image taken at the top of the CNT shows signatures of "odako" growth. (d-e) Variation in the height of VA-CNTs grown on ion beam damaged sapphire substrates. The substrates were prepared using different degrees of $\mathrm{Ar}^{+}$ion beam bombardment at variable dose with a fixed accelerated voltage and at variable accelerating voltages at a fixed dose. (f) SEM image of patterned VA-CNT growth via masked ion beam bombardment in selective regions of a sapphire substrate. Inset shows high mag. SEM image near the base of one patterned area. Figures a-b and d-f are reproduced from Ref. [33].

\section{Surface and structural characterization}

To analyze the catalysts used for VA-CNT growth on different damaged surfaces, $\mathrm{Ar}^{+}$ion damaged sapphire substrates were coated with an approximate $1 \mathrm{~nm}$ Fe catalyst film, as used for CNT growth, and then annealed in $\mathrm{H}_{2}$ ambient at 585 ${ }^{0} \mathrm{C}$ for $10 \mathrm{~min}$. Annealing of the catalyst film in $\mathrm{H}_{2}$ ambient induces de-wetting of the film via coarsening and subsurface diffusion into the porous catalyst support [23] and forms iron nanoparticles on top of the engineered sapphire surfaces. 
Figure 2a-b suggests that the Fe nanoparticles formed on surfaces prepared using a lower degree of $\mathrm{Ar}^{+}$ion damage are larger and isolated; while that formed on surfaces prepared using a higher degree of $\mathrm{Ar}^{+}$ion damage are smaller and dense. A heavily damaged surface, therefore, show smaller surface roughness (Figure 2d), higher particle density (Figure 2e) and hence greater catalytic activity compared to a lightly damaged one. The XPS surface analysis also reconfirms this trend. The measured increase in both the $\mathrm{O} / \mathrm{Al}$ atomic ratio (Figure 2f) and the $\mathrm{O} 1 \mathrm{~s}$ peak width (Figure $2 \mathrm{~g}$ ) with the increase in the degree ion damage suggests hydroxyl enrichment on the damaged sapphire surfaces that correlates to a higher degree of catalytic activity and hence longer lifetime for the Fe catalyst nanoparticles [32]. Surface analysis data collected using AFM and XPS, therefore, suggests non-stoichiometric sapphire surfaces with enriched hydroxyl groups after ion beam damage. The induced disorder enhances catalyst activity and lifetime and obviates the need to deposit an additional alumina layer for VA-CNT growth.
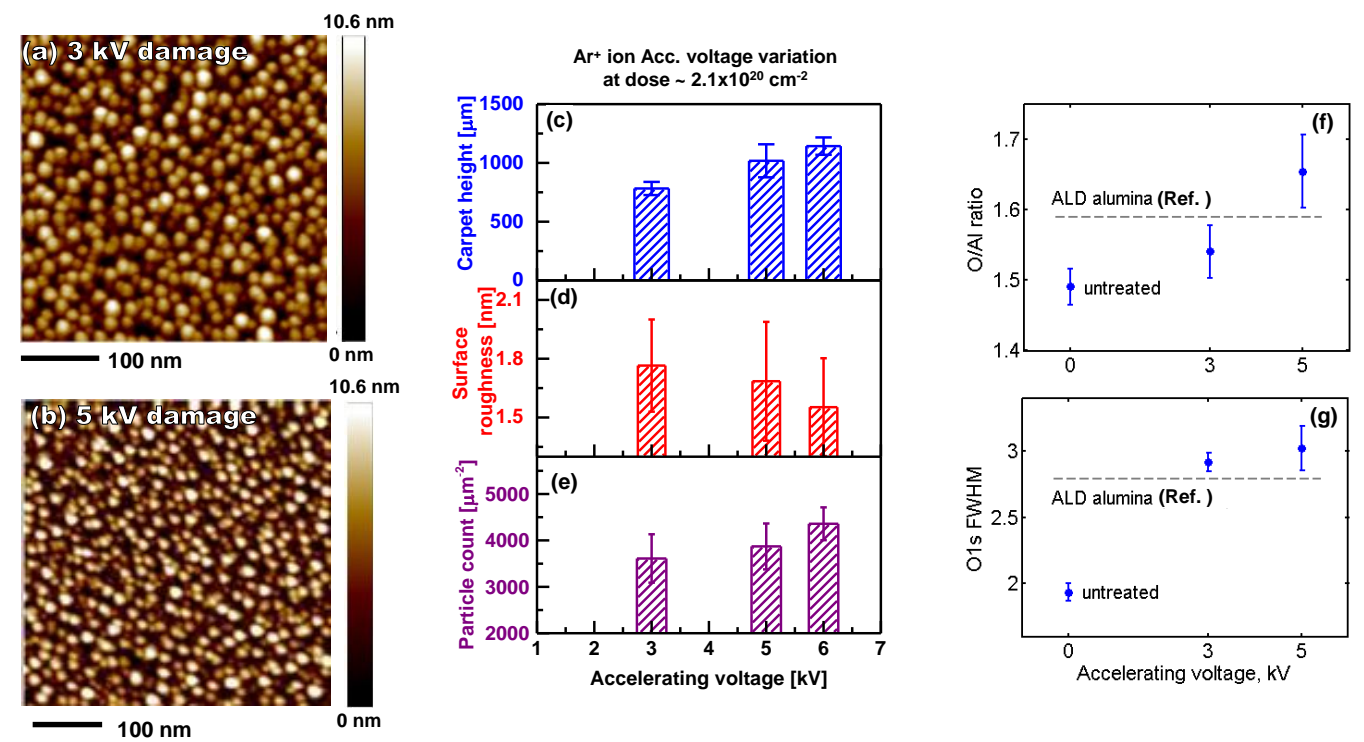

Figure 2: Summary of surface characterization techniques used to understand catalytic activity on damaged sapphire substrates: (a-b) AFM topography of Fe nanoparticles formed on sapphire surfaces prepared with different degrees of ion beam bombardment obtained by varying the acceleration voltage at a fixed dose of $2.1 \times 10^{20} \mathrm{~cm}^{-2}$. To form these nanoparticles, Fe film of $\sim 1 \mathrm{~nm}$ was coated on the surfaces followed by a 10 min hydrogen anneal at $585{ }^{\circ} \mathrm{C}$. (c-e) A comparison of the height of VA-CNTs grown on damaged substrates with the surface roughness and nanoparticle density obtained after $\mathrm{H}_{2}$ anneal of $1 \mathrm{~nm}$ Fe film using methods discussed above. Trends indicate that increasing damage via increase in ion energy induces longer VA-CNTs with smaller and denser particles. (f-g) The variation in the ratio of the $\mathrm{O}$ 1s peak area to the $\mathrm{Al} 2 \mathrm{p}$ XPS peak area (O/Al ratio) and FWHM for the $\mathrm{O}$ 1s peak with increasing degrees ion beam damage suggests higher surface activity for higher damage. Figures (c-f) are reproduced from Ref. [33].

As porosity of a substrate surface defines catalytic activity [23], we performed structural analysis using X-TEM and XRR. The X-TEM image of a sapphire substrate (Figure 3a) damaged using $3.8 \times 10^{20} \mathrm{Ar}^{+}$ions $/ \mathrm{cm}^{2}$ at an acceleration voltage of $5 \mathrm{kV}$ shows the presence of $\sim 13 \mathrm{~nm}$ thick damaged layer near the surface. Consistent with previous studies of ion implantation of different substrates [39-41], this damage layer consists of an epitaxially regrown amorphous layer at the top, which is followed by a porous, interfacial layer in between the amorphous and crystalline regions. The XRR analysis performed on sapphire substrates prepared using different degrees of ion beam damage also suggests porosity in damaged layers and a systematic change in damage depth with the increase in beam damage (table in Figure $3 \mathrm{~b}$ ). All these confirms that the porosity in the ion beam damaged surface allows more sub-surface diffusion of $\mathrm{Fe}$ into the catalyst support, results less Oswald ripening and hence enhances catalytic activity.

\section{CONCLUSION}

We have presented a novel way to control catalytic activity on an inert substrate via ion beam bombardment. The method expands the choice catalyst supports currently being used for VA-CNT growth. The contrast between the lack of growth on the pristine substrate and the VA-CNT growth in $\mathrm{Ar}^{+}$ion bombarded areas highlights the sharp difference in 
catalytic activity between an atomically perfect surface and an intentionally disrupted one. Applied microstructural and surface chemical characterization tools on the engineered catalyst supports validate our current understanding of Ostwald ripening, coarsening, and surface chemistry as defining mechanisms for VA-CNT growth.
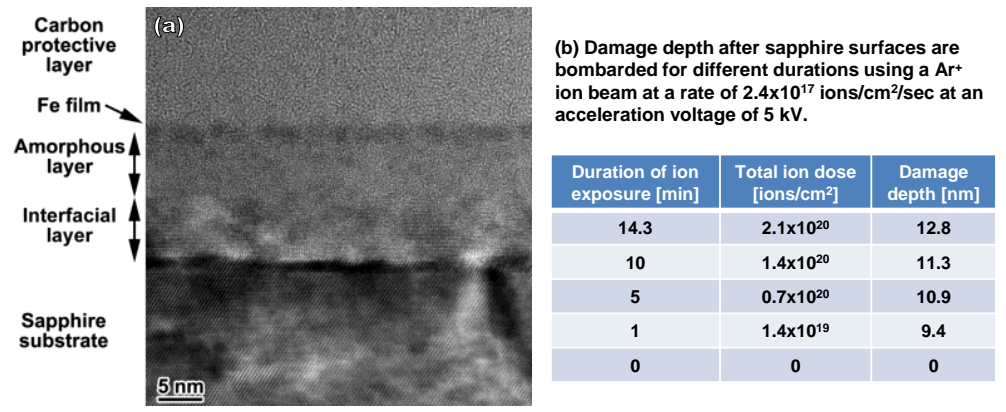

Figure 3: (a) X-TEM image of an ion beam damaged sapphire substrate. The substrate was damaged with $3.8 \times 10^{20} \mathrm{Ar}^{+}$ ions $/ \mathrm{cm}^{2}$ at an acceleration voltage of $5 \mathrm{kV}$ and then coated with a $\sim 1 \mathrm{~nm}$ Fe film [33]. (b) A summary of XRR data obtained by exposing damaged surfaces to small-angle X-ray. The damage depths are extracted from a fit to the XRR data obtained at different angles $\left(2 \theta=0.4-8^{\circ}\right)$.

\section{ACKNOWLEDGEMENT}

This work was supported by the Air Force Office of Scientific Research, under Contract No. FA 8650-09-D-5037. A part of the research was carried out at the Center for Functional Nanomaterials, Brookhaven National Laboratory, which is supported by the U.S. Department of Energy, Office of Basic Energy Sciences, under Contract No. DE-AC0298CH10886. A.E. Islam acknowledges support from the National Research Council Postdoctoral Fellowship program and S. Saber acknowledges support from the Purdue Electron Microscopy Consortium.

\section{REFERENCES}

[1] O. Breuer, and U. Sundararaj, "Big returns from small fibers: A review of polymer/carbon nanotube composites," Polymer Composites, 25(6), 630-645 (2004).

[2] I. M. De Rosa, F. Sarasini, M. S. Sarto et al., "EMC impact of advanced carbon fiber/carbon nanotube reinforced composites for next-generation aerospace applications," Ieee Transactions on Electromagnetic Compatibility, 50(3), 556-563 (2008).

[3] J. K. W. Sandler, J. E. Kirk, I. A. Kinloch et al., "Ultra-low electrical percolation threshold in carbon-nanotubeepoxy composites," Polymer, 44(19), 5893-5899 (2003).

[4] N. Behabtu, C. C. Young, D. E. Tsentalovich et al., "Strong, Light, Multifunctional Fibers of Carbon Nanotubes with Ultrahigh Conductivity," Science, 339(6116), 182-186 (2013).

[5] X. Wang, N. Behabtu, C. C. Young et al., "High-Ampacity Power Cables of Tightly-Packed and Aligned Carbon Nanotubes," Advanced Functional Materials, 24(21), 3241-3249 (2014).

[6] S. Esconjauregui, M. Fouquet, B. C. Bayer et al., "Growth of Ultrahigh Density Vertically Aligned Carbon Nanotube Forests for Interconnects," Acs Nano, 4(12), 7431-7436 (2010).

[7] Q. F. Pengfei, O. Vermesh, M. Grecu et al., "Toward large arrays of multiplex functionalized carbon nanotube sensors for highly sensitive and selective molecular detection," Nano Letters, 3(3), 347-351 (2003).

[8] P. G. Collins, K. Bradley, M. Ishigami et al., "Extreme oxygen sensitivity of electronic properties of carbon nanotubes," Science, 287(5459), 1801-1804 (2000).

[9] A. C. Dillon, "Carbon Nanotubes for Photoconversion and Electrical Energy Storage," Chemical Reviews, 110(11), 6856-6872 (2010).

[10] Q. Zhang, J.-Q. Huang, W.-Z. Qian et al., "The Road for Nanomaterials Industry: A Review of Carbon Nanotube Production, Post-Treatment, and Bulk Applications for Composites and Energy Storage," Small, 9(8), 1237-1265 (2013).

[11] P. Avouris, "Nanotube electronics - Electronics with carbon nanotubes," Physics World, 20(3), 40-45 (2007). 
[12] Q. Cao, S.-j. Han, G. S. Tulevski et al., "Arrays of single-walled carbon nanotubes with full surface coverage for high-performance electronics," Nature Nanotechnology, 8(3), 180-186 (2013).

[13] S. H. Jin, S. N. Dunham, J. Song et al., "Using nanoscale thermocapillary flows to create arrays of purely semiconducting single-walled carbon nanotubes," Nature Nanotechnology, 8(5), 347-355 (2013).

[14] K. Hasegawa, and S. Noda, "Millimeter-Tall Single-Walled Carbon Nanotubes Rapidly Grown with and without Water," Acs Nano, 5(2), 975-984 (2011).

[15] K. Hata, D. N. Futaba, K. Mizuno et al., "Water-assisted highly efficient synthesis of impurity-free single-waited carbon nanotubes," Science, 306(5700), 1362-1364 (2004).

[16] H. Kimura, J. Goto, S. Yasuda et al., "Unexpectedly High Yield Carbon Nanotube Synthesis from Low-Activity Carbon Feedstocks at High Concentrations," Acs Nano, 7(4), 3150-3157 (2013).

[17] S. Yasuda, D. N. Futaba, T. Yamada et al., "Improved and Large Area Single-Walled Carbon Nanotube Forest Growth by Controlling the Gas Flow Direction," Acs Nano, 3(12), 4164-4170 (2009).

[18] A. Naeemi, and J. D. Meindl, "Design and performance modeling for single-walled carbon nanotubes as local, semiglobal, and global interconnects in gigascale integrated systems," Ieee Transactions on Electron Devices, 54(1), 26-37 (2007).

[19] J. H. Hafner, M. J. Bronikowski, B. R. Azamian et al., "Catalytic growth of single-wall carbon nanotubes from metal particles," Chemical Physics Letters, 296(1-2), 195-202 (1998).

[20] D. Yuan, L. Ding, H. Chu et al., "Horizontally aligned single-walled carbon nanotube on quartz from a large variety of metal catalysts," Nano Letters, 8(8), 2576-2579 (2008).

[21] D. N. Futaba, K. Hata, T. Namai et al., " $84 \%$ Catalyst activity of water-assisted growth of single walled carbon nanotube forest characterization by a statistical and macroscopic approach," Journal of Physical Chemistry B, 110(15), 8035-8038 (2006).

[22] P. B. Amama, C. L. Pint, L. McJilton et al., "Role of Water in Super Growth of Single-Walled Carbon Nanotube Carpets," Nano Letters, 9(1), 44-49 (2009).

[23] P. B. Amama, C. L. Pint, S. M. Kim et al., "Influence of Alumina Type on the Evolution and Activity of AluminaSupported Fe Catalysts in Single-Walled Carbon Nanotube Carpet Growth," Acs Nano, 4(2), 895-904 (2010).

[24] P. B. Amama, C. L. Pint, F. Mirri et al., "Catalyst-support interactions and their influence in water-assisted carbon nanotube carpet growth," Carbon, 50(7), 2396-2406 (2012).

[25] S. M. Kim, C. L. Pint, P. B. Amama et al., "Catalyst and catalyst support morphology evolution in single-walled carbon nanotube supergrowth: Growth deceleration and termination," Journal of Materials Research, 25(10), 18751885 (2010).

[26] C. Mattevi, C. T. Wirth, S. Hofmann et al., "In-situ X-ray photoelectron spectroscopy study of catalyst-support interactions and growth of carbon nanotube forests," Journal of Physical Chemistry C, 112(32), 12207-12213 (2008).

[27] B. Wang, Y. Yang, L.-J. Li et al., "Effect of different catalyst supports on the (n,m) selective growth of singlewalled carbon nanotube from Co-Mo catalyst," Journal of Materials Science, 44(12), 3285-3295 (2009).

[28] S. M. Kim, C. L. Pint, P. B. Amama et al., "Understanding Growth Termination of Single-Walled Carbon Nanotube Carpets by Documenting the Evolution of Catalyst Morphology with the Transmission Electron Microscope," Microscopy and Microanalysis, 15, 1176-1177 (2009).

[29] S. M. Kim, C. L. Pint, P. B. Amama et al., "Evolution in Catalyst Morphology Leads to Carbon Nanotube Growth Termination," Journal of Physical Chemistry Letters, 1(6), 918-922 (2010).

[30] Y. Murakami, S. Chiashi, Y. Miyauchi et al., "Growth of vertically aligned single-walled carbon nanotube films on quartz substrates and their optical anisotropy," Chemical Physics Letters, 385(3-4), 298-303 (2004).

[31] H. Ohno, D. Takagi, K. Yamada et al., "Growth of vertically aligned single-walled carbon nanotubes on alumina and sapphire substrates," Japanese Journal of Applied Physics, 47(4), 1956-1960 (2008).

[32] P. B. Amama, S. A. Putnam, A. R. Barron et al., "Wetting behavior and activity of catalyst supports in carbon nanotube carpet growth," Nanoscale, 5(7), 2642-2646 (2013).

[33] A. E. Islam, P. Nikolaev, P. B. Amama et al., "Engineering the Activity and Lifetime of Heterogeneous Catalysts for Carbon Nanotube Growth via Substrate Ion Beam Bombardment," Nano Letters, 14(9), 4997-5003 (2014).

[34] S. Chakrabarti, H. Kume, L. Pan et al., "Number of walls controlled synthesis of millimeter-long vertically aligned brushlike carbon nanotubes," Journal of Physical Chemistry C, 111(5), 1929-1934 (2007).

[35] J.-Q. Huang, Q. Zhang, M.-Q. Zhao et al., "Patterning of hydrophobic three-dimensional carbon nanotube architectures by a pattern transfer approach," Nanoscale, 2(8), 1401-1404 (2010). 
[36] J. Huang, Q. Zhang, M. Zhao et al., "Process Intensification by CO2 for High Quality Carbon Nanotube Forest Growth: Double-Walled Carbon Nanotube Convexity or Single-Walled Carbon Nanotube Bowls?," Nano Research, 2(11), 872-881 (2009).

[37] C. L. Pint, N. T. Alvarez, and R. H. Hauge, "Odako Growth of Dense Arrays of Single-Walled Carbon Nanotubes Attached to Carbon Surfaces," Nano Research, 2(7), 526-534 (2009).

[38] Y. Yun, V. Shanov, Y. Tu et al., "Growth mechanism of long aligned multiwall carbon nanotube arrays by waterassisted chemical vapor deposition," Journal of Physical Chemistry B, 110(47), 23920-23925 (2006).

[39] J. Linnros, B. Svensson, and G. Holmen, "Ion-beam-induced Epitaxial Regrowth of Amorphous Layers in Silicon on Sapphire," Physical Review B, 30(7), 3629-3638 (1984).

[40] B. Canut, A. Benyagoub, G. Marest et al., "Swift-Uranium-ion-induced Damage in Sapphire," Physical Review B, 51(18), 12194-12201 (1995).

[41] I. Golecki, G. E. Chapman, S. S. Lau et al., "Ion-beam Induced Epitaxy of Silicon," Physics Letters A, 71(2-3), 267-269 (1979). 